\title{
SIT bei Insektengiftallergie - im Zweifelsfall lebenslänglich
}

\author{
Etwa 3\% der Deutschen reagieren allergisch gegen Bienen- oder \\ Wespengift. Wegen der Gefahr einer fatalen anaphylaktischen \\ Reaktion erhalten diese Patienten eine spezifische Immuntherapie \\ (SIT) - Risikopatienten sogar lebenslänglich.
}

\begin{abstract}
$\mathrm{D}$ ie diagnostischen Standardmethoden Pricktest und RAST bringen nicht alle Insektengiftallergien zutage, warnte Prof. Dr. Bernhard Przybilla, München. Dann zeigt manchmal der Basophilen-Aktivierungs- oder der Basophilen-Histaminfreisetzungstest das Problem. Darum spielt in jedem Fall die Anamnese eine wichtige Rolle bei der Evaluation. Niemals darf dagegen bei nicht hyposensibilisierten Patienten mit Verdacht auf Insektengiftallergie ein Stichprovokationstest vorgenommen werden. Besonders gefährdet sind nicht nur Imker und ihre Angehörigen, sondern alle, die häufig mit Insekten konfrontiert sind - vom Landwirt über die Bäckereiverkäuferin und den Gärtner bis zum Golfspieler und Motorradfahrer.
\end{abstract}

\section{Kontraindikationen beachten}

Liegen schwere Herz-Kreislauf-Erkrankungen vor, ist eine SIT mit Aeroallergenen kontraindiziert, mit Insektengift jedoch besonders wichtig. Die Notwendigkeit einer Umstellung der Behand- lung, z. B. bei Einnahme von $\beta$-Blockern, ist dann sorgfältig zu überprüfen. In der Schwangerschaft kann die SIT nicht begonnen, bei guter Verträglichkeit jedoch fortgeführt werden. Die SIT sollte vor einer Gravidität eingeleitet werden, da eine Anaphylaxie der Mutter das Ungeborene schwer schädigen kann. Wenn nötig, wird die Insektengift-SIT auch bei sehr jungen Kindern vorgenommen. Przybilla berichtete von der Hyposensibilisierung eines zweijährigen Kindes in seiner Klinik - unter sorgfältiger Überwachung.

Przybilla führt die Hymenopterengift-SIT im Rush-Verfahren durch und erreicht nach vier bis fünf Tagen die Erhaltungsdosis - klare Empfehlungen zur Wahl des Protokolls in der Steigerungsphase gibt es zwar nicht, das Ultra-RushVerfahren sollte aber nur unter Bereithaltung von besonderen Notfall- und Intensivmaßnahmen erfolgen. Zeigt sich mit einer Erhaltungsdosis von $100 \mu \mathrm{g}$ nach einem halben bis einem Jahr bei der Stichprovokation noch eine Reaktion - etwa bei einem Fünftel der Patienten ist dies zu erwarten -, wird die Dosis auf $200 \mu \mathrm{g}$ erhöht. Mit dieser Dosis kann nach Przybillas Erfahrung fast immer ein ausreichender Schutz erreicht werden.

\section{Absetzen individuell abwägen}

Die Langzeitwirkung der InsektengiftHyposensibilisierung ist noch nicht eindeutig zu beurteilen. Daher bleibe, erklärte Przybilla, das Absetzen der SIT eine individuelle Entscheidung und sollte dem Patienten nicht von vorneherein in Aussicht gestellt werden. Empfohlen wird eine mindestens drei bis fünf Jahre dauernde SIT. Dann kommt ein Absetzen infrage, wenn auf einen akzidentiellen Stich oder eine Stichprovokation keine Reaktion mehr erfolgt und die SIT ohne systemische Nebenwirkungen vertragen wurde. Bei Mastozytose ist in jedem Fall eine lebenslange SIT indiziert, ggf. auch bei anderen besonderen Risiken. An eine Mastozytose sollte bei jeder schweren Reaktion auf einen Bienen- oder Wespenstich gedacht und eine Tryptasebestimmung durchgeführt werden - etwa 25\% der schweren anaphylaktischen Reaktionen sind darauf zurück zu führen. Bei Mastozytose und Bienengiftallergie empfiehlt Przybilla, die SIT sofort mit einer höheren Erhaltungsdosis zu planen. $f k$

Przybilla B. Insektengiftallergie: Antworten auf häufige Fragen. 22. Fortbildungskongress „Fortschritte der Allergologie, Dermatologie, Pneumologie und Immunologie“, Davos, 6.-9. September 2006

\section{Die SLIT auf dem Prüfstand}

Für die sublinguale Immuntherapie liegen inzwischen zahlreiche Studien zur Wirksamkeit und Sicherheit vor. Dennoch fällt die Bewertung für die Praxis nicht leicht - zu viele Fragen sind noch offen, meint Priv.-Doz. Dr. Jörg Kleine-Tebbe aus Berlin.

E ine plazebokontrollierte Vergleichsstudie (Khinchi MS et al. Allergy 2004; 59: 45-53) konnte im Jahr 2004 zeigen, dass sowohl die sublinguale Immuntherapie (SLIT) als auch die subkutane Immuntherapie (SCIT) eine signi- fikante Symptomreduktion gegenüber einer Scheinbehandlung erzielen. KleineTebbe warnt aber davor, den nicht signifikanten Unterschied der Wirksamkeit als Beleg für eine Gleichwertigkeit beider Methoden zu werten - zu klein sei die
Probandenzahl in der Studie gewesen, meint er. Auch die Metaanalyse von 22 Studien der Cochrane-Collaboration (Wilson DR et al. Cochrane Database Syst Rev 2003; 2: CD002893) gebe kein klares Bild: Zwar weise die Analyse auf einen leichten bis moderaten Effekt der SLIT im Vergleich zu Plazebo hin, die einzelnen Studien zeigten aber eine sehr ausgeprägte Heterogenität. Eine gute Wirksamkeit sei am ehesten bei Erwachsenen, bei saisonalen Allergien und in kurzen Studien zu finden, resümierte der Berliner Allergologe. Es blieben aber noch viele Fragen offen: von der optimalen Dosis und Dauer der Behandlung über 